$11-2014$

\title{
Estimates and Forecasts of GARCH Model under Misspecified Probability Distributions: A Monte Carlo Simulation Approach
}

OlaOluwa S. Yaya

University of Ibadan, Ibadan, Nigeria, os.yaya@ui.edu.ng

Olusanya E. Olubusoye

University of Ibadan, Ibadan, Nigeria, busoye2001@yahoo.com

Oluwadare O. Ojo

Federal University of Technology Akure, Akure, Nigeria, daruu208075@yahoo.com

Follow this and additional works at: http://digitalcommons.wayne.edu/jmasm

Part of the Applied Statistics Commons, Social and Behavioral Sciences Commons, and the Statistical Theory Commons

\section{Recommended Citation}

Yaya, OlaOluwa S.; Olubusoye, Olusanya E.; and Ojo, Oluwadare O. (2014) "Estimates and Forecasts of GARCH Model under Misspecified Probability Distributions: A Monte Carlo Simulation Approach," Journal of Modern Applied Statistical Methods: Vol. 13 : Iss. 2 , Article 28.

DOI: $10.22237 /$ jmasm/1414816020

Available at: http://digitalcommons.wayne.edu/jmasm/vol13/iss2/28

This Regular Article is brought to you for free and open access by the Open Access Journals at DigitalCommons@WayneState. It has been accepted for inclusion in Journal of Modern Applied Statistical Methods by an authorized editor of DigitalCommons@WayneState. 


\title{
Estimates and Forecasts of GARCH Model under Misspecified Probability Distributions: A Monte Carlo Simulation Approach
}

\author{
OlaOluwa S. Yaya \\ University of Ibadan \\ Ibadan, Nigeria
}

\author{
Olusanya E. Olubusoye \\ University of Ibadan \\ Ibadan, Nigeria
}

\author{
Oluwadare O. Ojo \\ Federal University of \\ Technology Akure \\ Akure, Nigeria
}

\begin{abstract}
The effect of misspecification of correct sampling probability distribution of Generalized Autoregressive Conditionally Heteroscedastic (GARCH) processes is considered. The three assumed distributions are the normal, Student $t$, and generalized error distributions. The GARCH process is sampled using one of the distributions and the model is estimated based on the three distributions in each sample. Parameter estimates and forecast performance are used to judge the estimated model for performance. The AR-GARCHGED performed better on the three assumed distributions; even, when Student $t$ distribution is assumed, AR-GARCH-Student $t$ does not perform as the best model.
\end{abstract}

Keywords: Generalized Error Distribution, forecasts, GARCH, misspecification, specification

\section{Introduction}

Since the introduction of Generalized Autoregressive Conditional Heteroscedastic (GARCH) model of Bollerslev (1986), thousands of articles have been published applying the model on financial series. The model captures volatility in the market, and its distributional specification makes it special among other nonlinear time series models. The GARCH process exists on the assumption of Normal, Student $t$, and Generalized Error Distributions (GED). The Normal distribution is the usual assumption in any time series estimation, but due to the fact that the distribution of GARCH process is leptokurtic, Normal distribution was found to be in appropriate

OlaOluwa Yaya and Olusanya Olubusoye are part of the academic staff in the Department of Statistics, University of Ibadan.Email them at os.yaya@ui.edu.ng. and oe.olubusoye@ui.edu.ng. O. O. Ojo is a lecturer in the Department of Statistics, Federal University of Technology, Akure. Email him at daruu208075@yahoo.com. 


\section{ESTIMATES OF GARCH MODEL}

in capturing the tail behavior of the series. Bollerslev (1987) therefore proposed Student $t$ distribution to capture the long tail behavior of the process. Nelson (1991) proposed the GED distribution.

Apart from the real applications of GARCH models on financial series, there is need to study the effect of misspecifying the GARCH distributional assumptions during estimation. Articles are very scarce along this line of thought. Wang (2002) affirms that spurious and inefficient inference is expected when pure GARCH models are misspecified. This as well may affect the Quasi Maximum Likelihood Estimates (QMLEs) of the misspecified model. The QMLE of pure GARCH( $(1,1)$ models indicates that the ARCH parameter is small, GARCH parameter is close to unity and the sum of both parameters approaches unity as the sampling frequency increases (Engle and Bollerslev, 1986; Bollerslev and Engle, 1993; Baillie, Bollerslev and Mikkelsen, 1996; Ding and Granger, 1996; Andersen and Bollerslev, 1997, and Engle and Patton, 2001.) This fact is reflected in the Integrated GARCH (IGARCH) of Engle and Bollerslev (1986). A more recent paper by Jensen and Lange (2010) shows that in a GARCH $(1,1)$ model, the estimates of $\hat{\alpha}_{1}$ and $\hat{\beta}_{1}$ tend to zero and unity respectively as the sampling frequency increased, which is an IGARCH effect. This IGARCH effect is known for pure-GARCH processes. In a linear AR-GARCH or nonlinear AR-GARCH processes, IGARCH effect is not plausible. The present work considers AR-GARCH process, and therefore IGARCH effect may not be expected.

As tail distribution of the GARCH model is captured using the three distributions, and parameters estimated adjust accordingly, forecasts performances of the model are affected. Extensive Monte Carlo simulation was performed on the GARCH model using the three distributions.

\section{The GARCH $(1,1)$ model}

The GARCH $(1,1)$ model proposed in Bollerslev (1986) is

$$
\sigma_{t}^{2}=w+\alpha_{1} \varepsilon_{t-1}^{2}+\beta_{1} \sigma_{t-1}^{2}
$$

where $\varepsilon_{t}$ are the returns series of the financial asset; $\sigma_{t}$ is the volatility at time $t$ and $z_{t}$ gives the assumed distribution. The parameters, $\alpha_{1}$ and $\beta_{1}$ are conditioned as $w>0, \alpha_{1} \geq 0, \beta_{1} \geq 0$, and $\alpha_{1}+\beta_{1}<1$ in order to ensure stationarity of the whole

process (Bollerslev, 1986). This condition is establish by defining 
$k_{t}=\varepsilon_{t}^{2}-\sigma_{t}^{2}=\left(z_{t}^{2}-1\right) \sigma_{t}^{2}$ where $z_{t} \approx N(0,1)$. Using this in (1) results in the Autoregressive Moving Average (ARMA) representation

$$
\varepsilon_{t}^{2}=w+\left(\alpha_{1}+\beta_{1}\right) \varepsilon_{t-1}^{2}-\beta_{1} k_{t-1}+k_{t}
$$

where $k_{t}$ is serially uncorrelated with mean zero. Stationarity of the process is then ensured when the roots of $1-\alpha(1)-\beta(1)=1-\left(\alpha_{1}+\beta_{1}\right)=0$ lie outside the unit circle and this is not conditioned on time $t$ as it is measured directly from the parameters of the model. Hence it is expected that

$$
\left(\alpha_{1}+\beta_{1}\right)<1
$$

for existence of covariance stationary process. For the stationary process, the finite unconditional variance of $\varepsilon t$ is given by

$$
\sigma^{2}=\frac{w}{1-\alpha_{1}-\beta_{1}}
$$

\section{Kurtosis of GARCH $(1,1)$ model}

For any $\operatorname{GARCH}(\mathrm{p}, \mathrm{q})$ process, $E(z t)=0$ and $\operatorname{Var}\left(z_{t}\right)=1 . E\left(z_{t}^{3}\right)$ is the skewness and $E\left(z_{t}^{4}\right)$ gives the measure of skewness. Because the emphasis is on tail behaviour of GARCH residuals, the expression for the unconditional kurtosis is next derived.

Assuming that $E\left(\sigma_{t}^{2}\right)$ and $E\left(\sigma_{t}^{4}\right)$ exist, then it suffices to write

$$
E\left(z_{t}^{4}\right)=k_{z}+3
$$

because $E\left(\varepsilon_{t}\right)=0$;

$$
E\left(\varepsilon_{t}^{4}\right)=E\left(\sigma_{t}^{4}\right) E\left(z_{t}^{4}\right) \text { because } \varepsilon_{t}=\sigma_{t} z_{t} \text { and } \varepsilon_{t} \text { and } z_{t} \text { are independent }
$$




\section{ESTIMATES OF GARCH MODEL}

Then, squaring GARCH $(1,1)$ model,

$$
\sigma_{t}^{2}=w+\alpha_{1} \varepsilon_{t-1}^{2}+\beta_{1} \sigma_{t-1}^{2}
$$

gives

$$
\sigma_{t}^{2}=w^{2}+\alpha_{1}^{2} \varepsilon_{t-1}^{4}+\beta_{1}^{2} \sigma_{t-1}^{4}+2 w \alpha_{1} \varepsilon_{t-1}^{2}+2 w \beta_{1} \sigma_{t-1}^{2}+2 \alpha_{1} \beta_{1} \sigma_{t-1}^{2} \varepsilon_{t-1}^{2}
$$

Taking expectation of the resulting expansion, as well as applying the properties outlined above

$$
E\left(\sigma_{t}^{2}\right)=\frac{w^{2}\left(1+\alpha_{1}+\beta_{1}\right)}{\left[1-\left(\alpha_{1}+\beta_{1}\right)\right]\left[1-\alpha_{1}^{2}\left(k_{z}+2\right)-\left(\alpha_{1}+\beta_{1}\right)^{2}\right]}
$$

Using the relation $E\left(\varepsilon_{t}^{4}\right)=\left(k_{z}+3\right) E\left(\sigma_{t}^{4}\right)$ where $k_{z}$ is the excess kurtosis of $z$, then

$$
E\left(\varepsilon_{t}^{4}\right)=\frac{w^{2}\left(1+\alpha_{1}+\beta_{1}\right)\left(k_{z}+3\right)}{\left[1-\left(\alpha_{1}+\beta_{1}\right)\right]\left[1-\alpha_{1}^{2}\left(k_{z}+2\right)-\left(\alpha_{1}+\beta_{1}\right)^{2}\right]}
$$

Using the formula $k_{\varepsilon}=\frac{E\left(\varepsilon_{t}^{4}\right)}{\left[E\left(\varepsilon_{t}^{2}\right)\right]^{2}}-3$ for excess kurtosis and with the fact that $E\left(\varepsilon_{t}^{2}\right)=\frac{w}{1-\alpha_{1}-\beta_{1}}$ from the properties above,

$$
\begin{aligned}
k_{\varepsilon} & =\frac{w^{2}\left(1+\alpha_{1}+\beta_{1}\right)\left(k_{z}+3\right)\left[1-\left(\alpha_{1}+\beta_{1}\right)\right]^{2}}{w^{2}\left[1-\left(\alpha_{1}+\beta_{1}\right)\right]\left[1-\alpha_{1}^{2}\left(k_{z}+2\right)-\left(\alpha_{1}+\beta_{1}\right)^{2}\right]}-3 \\
& =\frac{\left(k_{z}+3\right)\left[1-\left(\alpha_{1}+\beta_{1}\right)^{2}\right]}{\left[1-2 \alpha_{1}^{2}-\left(\alpha_{1}+\beta_{1}\right)^{2}-k_{z}\left(\alpha_{1}^{2}\right)\right]}-3
\end{aligned}
$$


with normally distributed innovations $z, k_{z}=0$ and

$$
k_{\varepsilon}=\frac{6 \alpha_{1}^{2}}{1-2 \alpha_{1}^{2}-\left(\alpha_{1}+\beta_{1}\right)^{2}}
$$

with non-normally distributed innovations $z$, as in Student $t$ and GED,

$$
\begin{array}{r}
\operatorname{Var}\left(z_{t}\right)=E\left(z_{t}^{2}\right)=1 \text { and } k_{z}=\frac{E\left(z_{t}^{4}\right)}{\left[E\left(z_{t}^{2}\right)\right]^{2}}=E\left(z_{t}^{4}\right) \neq 0, \text { then } \\
k_{\varepsilon}=\frac{k_{z}-k_{z}\left(\alpha_{1}+\beta_{1}\right)^{2}+6 \alpha_{1}^{2}+2 k_{z} \alpha_{1}^{2}}{1-2 \alpha_{1}^{2}-\left(\alpha_{1}+\beta_{1}\right)^{2}-k_{z}\left(\alpha_{1}^{2}\right)}
\end{array}
$$

In these two cases, it is observed that $\alpha_{1}^{2}$ is important in determining the tail behavior of $\varepsilon_{t}$, because once $\alpha_{1}^{2}=0, k_{\varepsilon}=0$. Hence, $k_{\varepsilon}=k_{z}$ for the non-normally distributed case and it implies the similarity of the tail behaviors of both $\varepsilon_{t}$ and $z_{t}$

\section{Distributional Assumptions and Estimation}

For GARCH models, the unconditional distributions are always non normal, and this gives fatter tails than the normal distribution. In practice, $z_{t}$ is assumed to follow the normal distribution or non-normal distributions. These non-normal distributions have been proved to perform well in modeling the fatter tails (leptokurticity) observed in GARCH residuals. The non-normal distributions are the Student $t$ distribution proposed in Bollerslev (1987) and Generalized Error Distribution (GED) by Nelson (1991)

The standardized Normal distribution is

$$
f\left(z_{t}\right)=\frac{1}{\sqrt{2 \pi}} \exp \left(-\frac{1}{2} z_{t}^{2}\right),-\infty<z_{t}<\infty
$$

with the log likelihood function

$$
L\left(z_{t}\right)=-\frac{1}{2}\left[N \log (2 \pi)+\sum_{t=1}^{N} z_{t}^{2}\right]
$$




\section{ESTIMATES OF GARCH MODEL}

where $N$ is the sample size. The standardized Student $t$ distribution proposed in Bollerslev (1987) is given as

$$
f\left(z_{t}, v\right)=\Gamma((v+1) / 2)[\Gamma(v / 2)]^{-1}[\pi(v)]^{-1 / 2}\left(1+\frac{z_{t}^{2}}{v}\right)^{-(v+1) / 2},-\infty<z_{t}<\infty
$$

This distribution is symmetric around zero as it is observed in its specification with $v>2$. At $v=1$, the Student $t$ reduces to Cauchy distribution. At $2<v \leq 4$, its conditional kurtosis is less than 3, which means that the resulting tail effect is normal. For $v>4$, the kurtosis becomes $3(v-1)(v-4)^{-1}$, which is greater than 3 , hence the tail effect becomes non-normal distribution. As $v \rightarrow \infty$, the distribution converges to normal distribution. The log likelihood function of Student $t$ distribution is then simplified as

$$
L\left(z_{t}, v\right)=-\frac{1}{2}\left\{N \log \left(\frac{\pi(v-2) \Gamma(v / 2)^{2}}{\Gamma((v+1) / 2)^{2}}\right)+(v+1) \sum_{t=1}^{N} \log \left[1+\frac{z_{t}^{2}}{(v-1)}\right]\right\}
$$

The standardized GED proposed in Nelson (1991) is given as

$$
f\left(z_{t}, v\right)=2^{-1} v \Gamma\left(\frac{3}{v}\right)^{1 / 2}\left[\Gamma\left(\frac{1}{v}\right)^{3 / 2}\right]^{-1} \exp \left[-\left|z_{t}\right|^{v}\left\{\Gamma\left(\frac{3}{v}\right)\left[\Gamma\left(\frac{1}{v}\right)\right]^{-1}\right\}^{v / 2}\right]
$$

where $-\infty<z_{t}<\infty$ and $v>0$. The GED reduces to the standard normal distribution at $v=4$. At $0<v<2$, the distribution has thicker tail than the normal distribution, for example, at $v=1$ the distribution becomes a double exponential (Laplace) distribution. At $v>2$, the distribution of $z t$ has thinner tails than the normal distribution, for example, as $v$ tends to infinity, $z_{t}$ reduces to a uniform distribution on the interval $(-\sqrt{3}, \sqrt{3})$. The $\log$ likelihood of this distribution is then expanded as

$$
f\left(z_{t}, v\right)=2^{-1} v \Gamma\left(\frac{3}{v}\right)^{1 / 2}\left[\Gamma\left(\frac{1}{v}\right)^{3 / 2}\right]^{-1} \exp \left[-\left|z_{t}\right|^{v}\left\{\Gamma\left(\frac{3}{v}\right)\left[\Gamma\left(\frac{1}{v}\right)\right]^{-1}\right\}^{v / 2}\right]
$$


These likelihood functions are then estimated using the numerical derivatives based on the fact that GARCH models lack closed form estimation. Berndt, Hall, Hall and Hausman (BHHH) algorithm of Berndt, et al (1974) is then used. This algorithm is termed Gauss-Newton in general Nonlinear Least Squares (NLS) and BHHH in MLE estimation. Unlike some other derivatives, it uses only first derivatives of the likelihood function and computes a set of parameter values as

$$
\psi^{(i+1)}=\psi^{(i)}-\left(\sum_{t=1}^{N} \frac{\partial L\left(z_{t}, .\right)_{t}^{(i)}}{\partial \psi} \cdot \frac{\partial L\left(z_{t}, \cdot\right)_{t}^{(i)}}{\partial \psi}\right)^{-1} \frac{\partial L\left(z_{t}, .\right)_{N}^{(i)}}{\partial \psi}
$$

where $L(z t,$.$) is the likelihood function. The initial parameter set is given as \psi^{(0)}$ and the parameter set which maximize the likelihood function is denoted as $\psi^{(i+1)}$. The estimation of GARCH $(1,1)$ model with Student $t$ distribution and GED follow the usual Quasi Maximum Likelihood Estimation (QMLE) because normality assumption is violated in these cases.

Misspecification of distribution of GARCH model could lead to stationarity and explosion of the series in some points. Though standard errors will be consistent; the QML estimators $\tilde{\psi}^{(i+1)}$ are generally closed to the exact ML estimator $\hat{\psi}^{(i+1)}$ for symmetric GARCH distribution. For non-symmetric conditional distributions, both the asymptotic and finite sample loss in efficiency are quite large and parametric estimation approach are not applicable in this regard (Mills and Markellos, 2008).

\section{Forecasts Evaluation}

Forecast evaluation criteria considered are the Root Mean Squares Forecast Error (RMSFE), Mean Absolute Error (MAE), Mean Absolute Percentage Forecast Error (MAPFE) and Theil Inequality of Theil $(1961 ; 1966)$. The MSFE is defined as

$$
M S F E=\frac{1}{m} \sum_{t=1}^{m}\left(\hat{\sigma}_{t}^{2}-\sigma_{t}^{2}\right)^{2}
$$

where $\hat{\sigma}_{t}^{2}$ is the predicted in-sample conditional variances, and this depends on the scale of the variance series, $\sigma_{t}^{2}$. The square root of MSFE is the RMSFE 


\section{ESTIMATES OF GARCH MODEL}

$$
R M S F E=\sqrt{\frac{1}{m} \sum_{t=1}^{m}\left(\hat{\sigma}_{t}^{2}-\sigma_{t}^{2}\right)^{2}}
$$

The MAFE and MAPFE are obtained by taking the absolute differences of the predicted conditional volatilities and the observed volatilities as

$$
\begin{gathered}
M A F E=\frac{1}{m} \sum_{t=1}^{m}\left|\hat{\sigma}_{t}^{2}-\sigma_{t}^{2}\right| \\
M A P F E=100 \sum_{t=1}^{m}\left|\frac{\hat{\sigma}_{t}^{2}-\sigma_{t}^{2}}{\sigma_{t}^{2}}\right|
\end{gathered}
$$

The Theil inequality is given as

$$
T I=\frac{\sqrt{\frac{1}{m} \sum_{t=1}^{m}\left(\hat{\sigma}_{t}^{2}-\sigma_{t}^{2}\right)^{2}}}{\sqrt{\frac{1}{m} \sum_{t=1}^{m} \hat{\sigma}_{t}^{2}}+\sqrt{\frac{1}{m} \sum_{t=1}^{m} \sigma_{t}^{2}}}
$$

The inequality coefficient is time invariant and always lies between 0 and unity. The smaller these forecast evaluation criteria, the better the candidate model represent well the data.

\section{Monte Carlo Simulations}

The Monte Carlo experiment is set up using the AR(1)-GARCH(1,1) DGP

$$
y_{t}=0.15+0.5 y_{t-1}+\varepsilon_{t}, \sigma_{t}^{2}=0.02+0.25 \varepsilon_{t-1}^{2}+0.60 \sigma_{t-1}^{2}
$$

with the error distribution $\varepsilon_{t}=\sigma_{t} z_{t}$ where $z_{t}$ is assumed to follow Normal, Student $t$ and GED distributions. The parameters of the AR(1) and GARCH(1,1) models are set within the stationary region in order to avoid problems data explosion. The sample sizes $N$ are varied as 2000, 4000 and 6000 with in-sample forecasts generated as $25 \%$ of the data length. The results are then presented as Scenarios 1 
to 3 in Tables 1-6 below. Each Scenario gives results for parameter estimation, volatility, excess kurtosis and forecasts evaluations criteria.

\section{Scenario 1: When the true Distribution is Normal}

Tables 1 and 2 present the results when the GARCH processes are simulated based on Normal distribution assumption, and these processes are used to estimate the GARCH process based on Student $t$, GED and the same Normal distribution. The results in Table 1 show that the AR-GARCH parameter estimates, measures of volatility and kurtosis are not consistent with sample sizes. Both the AR and GARCH parameter estimates computed for Student $t$ distribution have larger biases in compared with that of Normal and GED distributions, even though excess kurtosis of the AR-GARCH-Student $t$ model is the smallest. Volatility of the ARGARCH-Student $t$ model is also observed to be higher than that of the Normal and GED distributions. The excess kurtosis of the AR-GARCH-Normal model was expected to be the smallest because the series is sampled from Normal distribution but this was not the case.

Looking at the results of the in-sample forecasts realized from the ARGARCH models as given in Table 2, the AR-GARCH-Normal and AR-GARCHGED model perform better than AR-GARCH-Student $t$ model on forecasts as given by the minimum values of the RMSPE and Theil inequality coefficients. The ARGARCH-GED is expected to realize better forecasts than AR-GARCH-Normal model.

Table 1. Model Parameter, Volatility and Kurtosis when GARCH processes are simulated based on Normal distribution assumption

\begin{tabular}{|c|c|c|c|c|c|c|c|c|c|}
\hline $\begin{array}{l}\text { Assumed } \\
\text { Distribution }\end{array}$ & Sample & $\begin{array}{c}\hat{\phi}_{0} \\
(0.1500)\end{array}$ & $\begin{array}{c}\hat{\phi}_{1} \\
(0.5000)\end{array}$ & $\underset{(0.0200)}{\hat{w}}$ & $\begin{array}{c}\hat{\alpha}_{1} \\
(0.2500)\end{array}$ & $\begin{array}{c}\hat{\beta}_{1} \\
(0.6000)\end{array}$ & $\begin{array}{c}\text { Persistence } \\
(0.8500)\end{array}$ & Volatility & $\begin{array}{c}\text { Exc. } \\
\text { Kurtosis }\end{array}$ \\
\hline & 2000 & 0.1480 & 0.4839 & 0.0169 & 0.2110 & 0.6596 & 0.8706 & 0.1306 & 1.6427 \\
\hline \multirow[t]{3}{*}{ Normal } & 4000 & 0.1518 & 0.4724 & 0.0173 & 0.2049 & 0.6590 & 0.8639 & 0.1271 & 1.8430 \\
\hline & 6000 & 0.1475 & 0.4750 & 0.0180 & 0.2052 & 0.6503 & 0.8555 & 0.1246 & 1.3275 \\
\hline & 2000 & 0.1462 & 0.4868 & 0.0820 & 0.1500 & 0.6000 & 0.7500 & 0.3280 & 0.2895 \\
\hline \multirow[t]{3}{*}{ Student $t$} & 4000 & 0.1471 & 0.4794 & 0.0794 & 0.1500 & 0.6000 & 0.7500 & 0.3176 & 1.8408 \\
\hline & 6000 & 0.1576 & 0.4916 & 0.0849 & 0.1500 & 0.6000 & 0.7500 & 0.3396 & 0.4501 \\
\hline & 2000 & 0.1499 & 0.4811 & 0.0160 & 0.2125 & 0.6831 & 0.8956 & 0.1533 & 2.3761 \\
\hline \multirow[t]{2}{*}{ GED } & 4000 & 0.1547 & 0.4662 & 0.0172 & 0.2106 & 0.6743 & 0.8849 & 0.1494 & 2.1683 \\
\hline & 6000 & 0.1495 & 0.4723 & 0.0185 & 0.2118 & 0.6612 & 0.8730 & 0.1457 & 1.7707 \\
\hline
\end{tabular}




\section{ESTIMATES OF GARCH MODEL}

Table 2. Forecast evaluation estimates when GARCH processes are simulated based on Normal distribution assumption

\begin{tabular}{cccccc}
$\begin{array}{c}\text { Assumed } \\
\text { Distribution }\end{array}$ & Sample & RMSPE & MAFE & MAPFE & Theil \\
\hline \multirow{3}{*}{ Normal } & 2000 & 0.0003 & 0.0112 & 48.0967 & 0.0289 \\
& 4000 & 0.0002 & 0.0104 & 44.8820 & 0.0257 \\
& 6000 & 0.0001 & 0.0101 & 49.5855 & 0.0287 \\
\hline \multirow{2}{*}{ Student $\boldsymbol{t}$} & 2000 & 0.0011 & 0.0464 & 500.3362 & 0.1270 \\
& 4000 & 0.0007 & 0.0437 & 529.5600 & 0.1242 \\
\hline \multirow{2}{*}{ GED } & 6000 & 0.0008 & 0.0602 & 503.3612 & 0.1363 \\
& 2000 & 0.0003 & 0.0110 & 50.1820 & 0.0276 \\
& 4000 & 0.0002 & 0.0102 & 47.7504 & 0.0246 \\
& 6000 & 0.0001 & 0.0099 & 53.1115 & 0.0276 \\
\hline
\end{tabular}

\section{Scenario 2: When the true Distribution is Student $t$}

Tables 3 and 4 present the results when the true GARCH distribution follows Student $t$. Here, the distinctions in the GARCH estimates can only be made using the persistence and unconditional volatility measures. The AR-GARCH-Student $t$ model still presents smallest persistence and highest volatility. The excess kurtosis of the AR-GARCH-Student $t$ model is the smallest followed by that of ARGARCH-Normal model.

Table 3. Model Parameter, Volatility and Kurtosis when the true GARCH distribution follows Student $t$

\begin{tabular}{cccccccccc}
$\begin{array}{c}\text { Assumed } \\
\text { Distribution }\end{array}$ & Sample & $\hat{\phi}_{0}$ & $\hat{\phi_{1}}$ & $\begin{array}{c}\hat{w} \\
(\mathbf{0 . 1 5 0 0 )}\end{array}$ & $\begin{array}{c}\hat{\alpha}_{1} \\
\mathbf{( 0 . 5 0 0 0 )}\end{array}$ & $\begin{array}{c}\hat{\beta}_{1} \\
\mathbf{( 0 . 0 2 0 )}\end{array}$ & $\begin{array}{c}\text { Persistence } \\
(\mathbf{0 . 8 5 0 0 )}\end{array}$ & $\begin{array}{c}\text { Volatility } \\
\text { Kurtosis }\end{array}$ \\
\hline \multirow{2}{*}{ Normal } & 2000 & 0.1408 & 0.5096 & 0.0232 & 0.2295 & 0.6105 & 0.8400 & 0.1450 & 2.5966 \\
& 4000 & 0.1478 & 0.4830 & 0.0237 & 0.2576 & 0.5762 & 0.8338 & 0.1426 & 4.0384 \\
& 6000 & 0.1497 & 0.4878 & 0.0219 & 0.2483 & 0.6032 & 0.8515 & 0.1475 & 3.7926 \\
\hline \multirow{2}{*}{ Student $t$} & 4000 & 0.1472 & 0.4829 & 0.0911 & 0.1500 & 0.6000 & 0.7500 & 0.3644 & 0.8526 \\
& 6000 & 0.1408 & 0.5114 & 0.0961 & 0.1500 & 0.6000 & 0.7500 & 0.3844 & 1.1013 \\
\hline \multirow{2}{*}{ GED } & 2000 & 0.1510 & 0.5165 & 0.0938 & 0.1500 & 0.6000 & 0.7500 & 0.3752 & 0.7336 \\
& 4000 & 0.1473 & 0.4792 & 0.0243 & 0.2620 & 0.5937 & 0.8557 & 0.1684 & 5.7359 \\
& 6000 & 0.1491 & 0.4890 & 0.0230 & 0.2585 & 0.6093 & 0.8678 & 0.1740 & 5.9655 \\
\hline
\end{tabular}




\section{YAYA ET AL.}

Table 4. Forecast evaluation estimates when the true GARCH distribution follows Student $t$

\begin{tabular}{cccccc}
$\begin{array}{c}\text { Assumed } \\
\text { Distribution }\end{array}$ & Sample & RMSPE & MAFE & MAPFE & Theil \\
\hline \multirow{3}{*}{ Normal } & 2000 & 0.0004 & 0.0145 & 63.7385 & 0.0332 \\
& 4000 & 0.0002 & 0.0131 & 62.2680 & 0.0324 \\
& 6000 & 0.0003 & 0.0247 & 63.1032 & 0.0392 \\
\hline \multirow{2}{*}{ Student $\boldsymbol{t}$} & 2000 & 0.0015 & 0.0627 & 635.1114 & 0.1552 \\
& 4000 & 0.0010 & 0.0574 & 611.0665 & 0.1471 \\
& 6000 & 0.0009 & 0.0678 & 645.0181 & 0.1582 \\
\hline \multirow{2}{*}{ GED } & 2000 & 0.0004 & 0.0156 & 76.3588 & 0.0355 \\
& 4000 & 0.0002 & 0.0132 & 68.0163 & 0.0321 \\
& 6000 & 0.0004 & 0.0253 & 70.6578 & 0.0396 \\
\hline
\end{tabular}

In terms of forecasts, the AR-GARCH-Student $t$ model is the worst, even though the DGP is realized from the same probability distribution. The forecast performances of AR-GARCH-Normal and AR-GARCH-GED seem not different from each other as indicated by the forecast evaluation estimates.

\section{Scenario 3: When the true Distribution is GED}

Table 5 and 6 present the results when the true GARCH distribution is GED. In Table 5, in the $\operatorname{AR}(1)$ estimates, the estimates for the constant $\hat{\phi}_{0}$ are all consistent with sample sizes when the three probability distributions are assumed. The autoregressive parameters $\hat{\phi}_{1}$ are not consistent with sample sizes. The GARCH parameter estimates computed for Student $t$ distribution are the same to that of Table 1 and 3 while the AR(1) parameter are different in the two results. The Student $t$ distribution assumption of GARCH model still presents model estimates with highest volatility but with lowest persistence of this volatility. Misspecifying GED for Student $t$ distribution here also caused the excess kurtosis to be negative in AR-GARCH-Student $t$ model and this is a very spurious case.

The forecast evaluation results of the model estimates follow in In Table 6. Starting with the AR(1)-GARCH $(1,1)-$ Student $t$ model, the model is the worst in terms of forecasts because it presents the highest RMSPE, MAPE, MAPFE and Theil inequality coefficient. The best model is AR(1)- GARCH(1,1)-GED model, and this is expected because the DGP assumed GED initially. The performance of 


\section{ESTIMATES OF GARCH MODEL}

AR(1)-GARCH(1,1)-Normal in terms of forecast is very close to that of AR(1)-GARCH(1,1)-GED model.

Table 5. Model Parameter, Volatility and Kurtosis when the true GARCH distribution is GED

\begin{tabular}{cccccccccc}
$\begin{array}{c}\text { Assumed } \\
\text { Distribution }\end{array}$ & Sample & $\begin{array}{c}\hat{\phi}_{0} \\
(\mathbf{0 . 1 5 0 0 )}\end{array}$ & $\begin{array}{c}\hat{\phi}_{1} \\
\mathbf{( 0 . 5 0 0 0 )}\end{array}$ & $\begin{array}{c}\hat{w} \\
(\mathbf{0 . 0 2 0 0 )}\end{array}$ & $\begin{array}{c}\hat{\alpha}_{1} \\
(\mathbf{0 . 2 5 0 0 )}\end{array}$ & $\begin{array}{c}\hat{\beta}_{1} \\
(\mathbf{0 . 6 0 0 0}\end{array}$ & $\begin{array}{c}\text { Persistence } \\
(\mathbf{0 . 8 5 0 0 )}\end{array}$ & Volatility & $\begin{array}{c}\text { Exc. } \\
\text { Kurtosis }\end{array}$ \\
\hline \multirow{2}{*}{ Normal } & 2000 & 0.1409 & 0.5120 & 0.0219 & 0.2260 & 0.6112 & 0.8372 & 0.1345 & 0.1984 \\
& 4000 & 0.1478 & 0.4848 & 0.0236 & 0.2576 & 0.5639 & 0.8215 & 0.1322 & 0.4768 \\
& 6000 & 0.1497 & 0.4880 & 0.0218 & 0.2465 & 0.5932 & 0.8397 & 0.1360 & 0.3859 \\
\hline \multirow{2}{*}{ Student $t \boldsymbol{t}$} & 2000 & 0.1373 & 0.5192 & 0.0877 & 0.1500 & 0.6000 & 0.7500 & 0.3508 & -0.3883 \\
& 4000 & 0.1469 & 0.4863 & 0.0854 & 0.1500 & 0.6000 & 0.7500 & 0.3416 & -0.3554 \\
& 6000 & 0.1500 & 0.4860 & 0.0875 & 0.1500 & 0.6000 & 0.7500 & 0.3500 & -0.4099 \\
\hline \multirow{2}{*}{ GED } & 2000 & 0.1424 & 0.5024 & 0.0225 & 0.2284 & 0.6031 & 0.8315 & 0.1335 & 0.1874 \\
& 4000 & 0.1481 & 0.4834 & 0.0228 & 0.2493 & 0.5763 & 0.8256 & 0.1307 & 0.4139 \\
& 6000 & 0.1498 & 0.4900 & 0.0214 & 0.2430 & 0.5973 & 0.8403 & 0.1340 & 0.3518 \\
\hline
\end{tabular}

Table 6. Forecast evaluation estimates when the true GARCH distribution is GED

\begin{tabular}{cccccc}
$\begin{array}{c}\text { Assumed } \\
\text { Distribution }\end{array}$ & Sample & RMSPE & MAFE & MAPFE & Theil \\
\hline \multirow{2}{*}{ Normal } & 2000 & 0.0002 & 0.0099 & 48.9918 & 0.0280 \\
& 4000 & 0.0002 & 0.0100 & 50.8079 & 0.0292 \\
& 6000 & 0.0002 & 0.0142 & 50.9182 & 0.0325 \\
\hline \multirow{2}{*}{ Student $t$} & 2000 & 0.0013 & 0.0529 & 521.4746 & 0.1396 \\
& 4000 & 0.0008 & 0.0496 & 499.5437 & 0.1329 \\
& 6000 & 0.0008 & 0.0547 & 494.8839 & 0.1379 \\
\hline \multirow{2}{*}{ GED } & 2000 & 0.0002 & 0.0100 & 49.5704 & 0.0284 \\
& 4000 & 0.0002 & 0.0097 & 48.9723 & 0.0284 \\
\hline
\end{tabular}




\section{Conclusion}

The misspecification of GARCH probability distribution functions were considered. These are the Normal, Student $t$ and Generalized Error Distributions (GED). The estimation convergence time varied based on the distribution and the set sample sizes. When a Normal distribution was assumed, the AR-GARCH-GED seemed to perform marginally better than AR-GARCH-Normal model in terms of forecasts as revealed in the estimates of the Theil inequality. Though, the AR-GARCH-Normal was the best model here in terms of parameter estimates, and this was expected because the DGP assumed Normal distribution initially. With the assumption of Student $t$ distribution in the DGP, the forecast performance of

the models computed with Normal distribution and GED reduced and these still presented better models than the corresponding AR-GARCH-Student $t$ model. Similar results were obtained when the DGP assumed GED.

It was also observed that all the results obtained, particularly the parameter estimates were not consistent with sample sizes. These are expected because volatility came into play. In empirical modeling research like this, interest should either lie in the behavior of the volatility-assuming a probability distribution which will give us the best volatility measurement-or in the forecasts. The best GARCH model may not actually produce the best forecast estimates and probability distributions have effect on the tail distribution of the innovations. This work can be replicated using higher order of the model, and in that case, more sophisticated software is recommended for the simulation in order to avoid convergence problems.

\section{References}

Andersen, T. G., \& Bollerslev, T. (1997). Intraday periodicity and volatility persistence in financial markets. Journal of Empirical Finance, 4, 115-158.

Baillie, R.T., Bollerslev, T., \& Mikkelsen, H. O. (1996). Fractional integrated generalized autoregressive conditional heteroscedasticity, Journal of Econometrics, 74, 3-30.

Berndt, E., Hall, B., Hall, R., \& Hausman, J. (1974). Estimation and inference in nonlinear structural models. Annals of Economic and Social Measurement, 3, 653-665.

Bollerslev, T. (1986). Generalized Autoregressive Conditional Heteroscedasticity. Journal of Econometrics, 31, 307-327. 


\section{ESTIMATES OF GARCH MODEL}

Bollerslev, T. (1987). A conditional heteroscedastic time series model for speculative prices and rates of return. Review of Economics and Statistics, 69, 542-547.

Bollerslev, T., \& Engle, R. F. (1993). Common persistence in conditional variances. Econometrica, 61, 167-186.

Ding, Z., \& Granger, C. W. J. (1996), Modelling volatility persistence of speculative returns: a new approach. Journal of Econometrics, 73, 185-215.

Engle, R. F., \& Bollerslev, T. (1986). Modeling the persistence of conditional variances. Econometric Reviews, 5, 1-50.

Engle, R. F., \& Patton, A. J. (2001). What good is a volatility model? Quantitative Finance, 1, 237-245.

Jensen, A. T., \& Lange, T. (2010). On convergence of the QMLE for misspecified GARCH models. Journal of Time Series Econometrics, 2(1), 1-31.

Mills, T. C., \& Markellos, R. (2008). The Econometric Modelling of Financial Time Series (3rd ed.). Cambridge, UK: Cambridge University Press.

Nelson, D. B. (1991). Conditional heteroscedasticity in asset returns: A new approach. Econometrica, 59, 347-370.

Theil, H. (1961). Economic forecasts and policy. Amsterdam: NorthHolland.

Theil, H. (1966). Applied Economic forecasting. Amsterdam: North-Holland

Wang, Y. (2002), Asymptotic nonequivalence of GARCH models and diffusions. Annals of Statistics, 30(3), 754-783. 